\title{
BOUND ON THE AMPLITUDE OF THE EARTH'S FREE CORE-NUTATION
}

\author{
T. A. Herring, C. R. Gwinn, B.A. Buffett, and I. I. Shapiro \\ Harvard-Smithsonian Center for Astrophysics \\ 60 Garden Street \\ Cambridge, MA. 02138
}

\begin{abstract}
We analyzed six years of very-long-baseline interferometry (VLBI) data and determined corrections to the coefficients of the seven terms with the largest amplitudes in the IAU 1980 nutation series. Our analysis yields results consistent with earlier analyses of smaller sets of VLBI data, within the uncertainties of the latter. Here, we restrict discussion to the freely excited core-nutation or "free core-nutation" (FCN). Our analysis yields an estimate of $0.33 \pm 0.12$ mas for an assumed constant amplitude of the FCN, which allows us to place an upper bound on it of 0.6 mas ( $99.5 \%$ confidence limit). We also studied possible temporal variations of the complex amplitude of the FCN by modeling it as a stochastic process with a white noise excitation. We detected no statistically significant variations of this amplitude for the six-year interval spanned by the VLBI data. However, in the neighborhood of one cycle per day, the power spectral density of the atmospheric surface loading is estimated from global weather data to be $0.24\left(\mathrm{~g} \mathrm{~cm}^{-2}\right)^{2}$ day, about five times larger than the largest such power spectral density that would be consistent with the upper bound on the amplitude of the FCN placed by the VLBI data. Thus, we conclude that this estimate is too high and that, if the FCN were excited by surface loads with frequencies near one cycle per day, then the power spectral density of these loads must be $<0.06\left(\mathrm{~g} \mathrm{~cm}^{-2}\right)^{2}$ day ( $99.9 \%$ confidence limit).
\end{abstract}

\section{INTRODUCTION}

The recent investigations of the nutations of the earth using VLBI data (Herring et al., 1983; Gwinn et al., 1984; Herring et al., 1985; Eubanks et al., 1985; and Herring et al., 1986) have all disclosed corrections to the coefficients of the terms in the IAU 1980 nutation series corresponding to the retrograde annual nutation and the prograde semiannual nutation. These corrections can be partially explained by changing the resonance frequency (in a frame rotating with the earth) of the "core nutation" from $-\left(1+\frac{1}{460}\right)$ cycles per sidereal day (cpsd) to $-\left(1+\frac{1}{433}\right)$ cpsd (Gwinn et al.,1986). Other phenomena contributing significantly to the corrections might be ocean tides (Wahr and Sasao, 1981) and the anelasticity of the earth's mantle (Wahr and Bergen, private communication, 1986). These latter two effects are not, however, nearly large enough by themselves to explain the $\approx 2$ mas correction to the amplitude of the retrograde annual nutation. These small corrections aside, the overall agreement between the VLBI results and the IAU 1980 nutation series is evidence for the existence of the predicted effect of the core-nutation resonance on the earth's rotation: this resonance contributes as much as 17 mas to 
the nutation series, in particular to the semiannual term. Nonetheless, the free excitation of this mode has never been unambiguously detected. Prior analyses of VLBI data have placed only upper bounds of under 1 mas on the amplitude of this free core nutation (FCN) [Herring et al., 1985; Eubanks et al., 1985; and Herring et al., 1986].

In this paper, we examine the FCN in more detail. In particular, we investigate the possibility that our single ("average") estimate of the amplitude of the FCN may be significantly less than the maximum value reached by this amplitude during the six-year interval spanned by the VLBI data, due to large fluctuations in this (complex) amplitude during this interval. This situation could arise if the FCN were heavily damped and highly excited. An estimated lower bound on the damping time, combined with an independent estimate of the expected amplitude of the FCN can be used to quantify statistically the possible fluctuations in this amplitude. We can obtain a lower bound for the damping time from the VLBI estimate of the amplitude of the out-of-phase component of the retrograde annual nutation. An estimate of the expected amplitude of the excitation of the FCN can be obtained from other geophysical data. In particular, we take the expected amplitude of the FCN from the magnitude obtained by Eubanks et al.(1985) of the power spectral density, at nearly diurnal periods, of the $P_{21}$ component of the atmospheric pressure loading of the earth's surface (see Wahr and Sasao (1981) for normalization of spherical harmonics).

\section{DATA ANALYSIS TECHNIQUE}

The techniques we have used to estimate corrections to the IAU 1980 nutation series have been discussed in Herring et al.(1985) and Herring et al.(1986). For each VLBI observing session (typically of 24 hours duration), we estimate corrections, $\delta \Delta \psi$ and $\delta \Delta \epsilon$, to the nutation angles computed from the IAU 1980 nutation series. In a post-processing operation, we use these nutation-angle corrections to estimate corrections to the coefficients of certain terms in the nutation series. The analysis used in this paper is similar to our previous analyses except that here we allow our model of the complex amplitude of the FCN to vary stochastically during the interval spanned by the data set. A Kalman filter (see, e.g., Liebelt, 1967) was used to estimate simultaneously the time-dependent complex amplitude of the FCN, and the corrections to the coefficients of selected terms in the IAU 1980 nutation series. was

The stochastic model used to represent the complex amplitude of the FCN

$$
\tilde{\zeta}(t+\Delta t)=\tilde{\zeta}(t) e^{-\alpha \Delta t}+\delta \tilde{\zeta}(t)
$$

where, respectively, $\tilde{\zeta}(t)$ and $\tilde{\zeta}(t+\Delta t)$ are the complex values of the FCN at times $t$ and $t+\Delta t ; \alpha(>0)$ is the inverse damping time for the FCN and stems from the imaginary, or dissipative, part of the FCN resonance frequency; and $\delta \tilde{\zeta}(t)$ is the complex excitation, integrated over the interval $t$ to $t+\Delta t\left(\Delta t \ll \alpha^{-1}\right)$. The corresponding contributions of the FCN to the nutation angles are

$$
\delta \Delta \epsilon_{F C N}(t)=-\zeta_{r}(t) \cos \left(\left(\Omega+\omega_{F C N}\right) t\right)-\zeta_{i}(t) \sin \left(\left(\Omega+\omega_{F C N}\right) t\right)
$$

and

$$
\delta \Delta \psi_{F C N}(t) \sin \epsilon_{\circ}=-\zeta_{r}(t) \sin \left(\left(\Omega+\omega_{F C N}\right) t\right)+\zeta_{i}(t) \cos \left(\left(\Omega+\omega_{F C N}\right) t\right),
$$

where, respectively, $\zeta_{r}(t)$ and $\zeta_{i}(t)$ are the real and imaginary parts of $\tilde{s}(t)=$ $\zeta_{r}(t)-2 \zeta_{i}(t) ; t$ is sidereal time (measured for convenience from the epoch J2000); $\Omega$ 
is the earth's rotation rate; and $\omega_{F C N}$ is the real component of the FCN frequency. To apply a Kalman filter to the estimates of $\delta \Delta \epsilon(t)$ and $\delta \Delta \psi(t)$ obtained from the VLBI data, we need to establish the statistical properties of the excitation of the FCN. However, we do not know the excitation mechanism for the FCN. The most efficient mechanism seems to be through surface loading (Sasao and Wahr, 1981). But, very little is known about the properties of non-tidal surface loading with periods near one day. Likely candidates for the excitation are loads from atmospheric pressure variations, and, possibly, from sea level changes (Sasao and Wahr, 1981). Since we know so little about the spectra of these excitations, we will make the simple assumption that all of them have white-noise spectra for periods near one day. We will characterize the amplitude of the white-noise excitation by its variance, $\sigma_{s}^{2}=\left\langle\delta \tilde{\xi}(t) \delta \tilde{\zeta}^{*}(t)\right\rangle$, where ${ }^{*}$ denotes complex conjugation, and \langle\rangle denotes expectation.

The magnitude of $\sigma_{\zeta}^{2}$ can be established by balancing the power input into the FCN by the excitation with that dissipated. From equation (1), we have for steady-state conditions (assuming $\alpha \Delta t \ll 1$ ):

$$
\sigma_{\zeta}^{2}=2\left\langle\tilde{\zeta} \tilde{\zeta}^{*}\right\rangle \alpha \Delta t .
$$

Therefore, if we know $\alpha$ and the expectation of the square of the magnitude of the (integrated) complex excitation of the FCN, we can compute the variance of the white-noise excitation to be used in the Kalman filter.

\section{RESULTS}

The VLBI data we have analyzed are from 370 observing sessions carried out by the NASA Crustal Dynamics Project and the NGS IRIS program between July 1980 and August 1986. This data set is an extension of those discussed in Herring et al.(1985) and Herring et al.(1986). In Table 1, we give the estimates of the amplitudes of the circular nutations that can be resolved with the limited temporal range of the VLBI data. The values for $\delta \Delta \epsilon$ and $\delta \Delta \psi \sin \epsilon_{\circ}$ we used to obtain these amplitude estimates are shown in Figure 1 . These amplitude results are based on the assumption that the amplitude of the FCN was constant for the six years spanned by the data set, i.e. that $\sigma_{\varsigma}^{2}=0$, whence the Kalman filter solution reduces to conventional weighted-least squares. In this solution, we not only estimated the amplitude of the FCN, but also the amplitude of a prograde ("control") term with the frequency $-\left(1-\frac{1}{433}\right)$ cpsd. Since no resonance is thought to exist for any such prograde term, any signal at this frequency is probably due to noise or to model deficiencies, and could be used as a measure of the uncertainty affecting the estimate of the FCN amplitude. The resultant estimates of the amplitudes of the FCN and the prograde terms are, respectively, $0.33 \pm 0.12$ and $0.04 \pm 0.12$ mas.

The amplitude of the FCN can also be estimated from other data. Eubanks et al. (1985) have estimated from global weather data that the power spectraldensity (PSD) of the $P_{21}$ component of the atmospheric pressure field is 0.24 (gm $\left.\mathrm{cm}^{-2}\right)^{2}$ day. (No uncertainty for this value is given by the authors. However, the estimate is stated to be "conservative.") This PSD, combined with the lower bound for damping time deduced from the VLBI data (see below), suggests that the FCN amplitude should be $\geq 0.8$ mas (see Sasao and Wahr, 1981 for computation methods). From this lower bound for the FCN amplitude, and the upper bound for $\alpha$ obtained from the out-of-phase component of the retrograde annual nutation, we estimate a lower bound for $\sigma_{\varsigma}^{2}$. Taking $\alpha$ to be $\leq 2 \pi \times 3.3 \times 10^{-5} \mathrm{~d}^{-1}(99.5 \%$ confidence limit) [see Gwinn et al.(1986) for computation method], we find $\sigma_{\varsigma}^{2} \geq$ $5.8 \times 10^{-4} \Delta t$ mas $^{2}$, where $\Delta t$ is in days. This lower bound for $\sigma_{\varsigma}^{2}$ indicates 
TABLE 1. Estimates of Corrections to Amplitudes of Circular Nutations of IAU 1980 Nutation Series (Wahr, 1981).

\begin{tabular}{cccc}
\hline & & \multicolumn{2}{c}{ Corrections $^{*}$} \\
\cline { 3 - 4 } $\begin{array}{c}\text { Period } \\
\text { in inertial } \\
\text { space, days }\end{array}$ & $\begin{array}{c}\text { Observed } \\
\text { Amplitude, } \\
\text { mas }\end{array}$ & $\begin{array}{c}\text { In-Phase } \\
\delta a_{r}, \\
\text { mas }\end{array}$ & $\begin{array}{c}\text { Out-of-Phase } \\
\delta a_{i}, \\
\text { mas }\end{array}$ \\
\hline 182.6 prograde & -548.63 & 0.44 & -0.41 \\
182.6 retrograde & -24.61 & -0.09 & -0.07 \\
13.7 prograde & -94.43 & -0.35 & 0.01 \\
13.7 retrograde & -3.64 & -0.02 & 0.04 \\
365.3 prograde & -35.73 & 0.07 & 0.15 \\
365.3 retrograde & -3.12 & -2.06 & 0.33 \\
27.6 prograde & 14.52 & 0.00 & 0.06 \\
27.6 retrograde & -13.78 & 0.04 & 0.00 \\
121.7 prograde & -21.58 & -0.10 & -0.03 \\
121.7 retrograde & -0.96 & -0.05 & -0.04 \\
9.1 prograde & -12.52 & -0.09 & 0.00 \\
9.1 retrograde & -0.45 & 0.01 & -0.02 \\
31.8 prograde & 3.17 & -0.03 & -0.03 \\
31.8 retrograde & -3.02 & 0.07 & -0.01 \\
433.2 prograde & $0.04 \dagger$ & -0.03 & -0.02 \\
433.2 retrograde FCN & $0.33 \dagger$ & 0.32 & -0.07 \\
\hline
\end{tabular}

*The standard deviation of each correction is estimated to be 0.10 mas, except for those of the annual and 433-day terms whose standard deviations are estimated to be 0.12 mas. The standard deviations are obtained using the techniques described in Herring et al. (1986), with the root-sum-squares addition of a contribution of 0.07 mas to account for (i) the effects of the truncation of the IAU 1980 nutation series to the nearest 0.1 mas, (ii) the possible effects of atmospheric excitation of the nutation, and (iii) the effects of ocean and earth tide modeling errors in the analysis of the VLBI data. See also Herring et al. (1986) for definitions of the quantities listed.

†The amplitudes of these terms are the root-sum-squares of the amplitudes of the real and imaginary components.

that the complex amplitude of the FCN would have a variance $>(0.5 \text { mas } / \text { year })^{2}$. (Although formally this estimate of the variance for the change in the complex amplitude of the FCN per year is a lower bound (due to our use of bounds in its calculation), our model probably predicts too much variation because of the assumed white-noise excitation which has a constant power-density, independent of frequency.) The VLBI estimates of the changes in both the FCN and the prograde signal are shown in Figure 2. The particular paths followed by each of these signals are not important. The important point is the absence of evidence that the single ("average") estimate of the amplitude of the FCN obtained from the VLBI data is greatly diminished by fluctuation in the complex amplitude of the FCN during the six-year interval spanned by the VLBI data. We also note that the average value of the amplitude of the FCN is consistent with our previous single estimate, and much smaller than the bound implied from the atmosphericpressure data.

The results given in Table 1 and Figure 2 indicate that the amplitude of the FCN is no larger than 0.6 mas ( $99.5 \%$ confidence limit). The VLBI upper bound for the FCN amplitude thus suggests that the PSD of the excitation is no more than $0.06\left(\mathrm{gm} \mathrm{cm}^{-2}\right)^{2}$ day $(99.9 \%$ confidence limit), about five times smaller than that calculated by Eubanks et al. Since we have used an upper bound for the FCN amplitude and a lower bound for the damping, the actual PSD of the excitation 


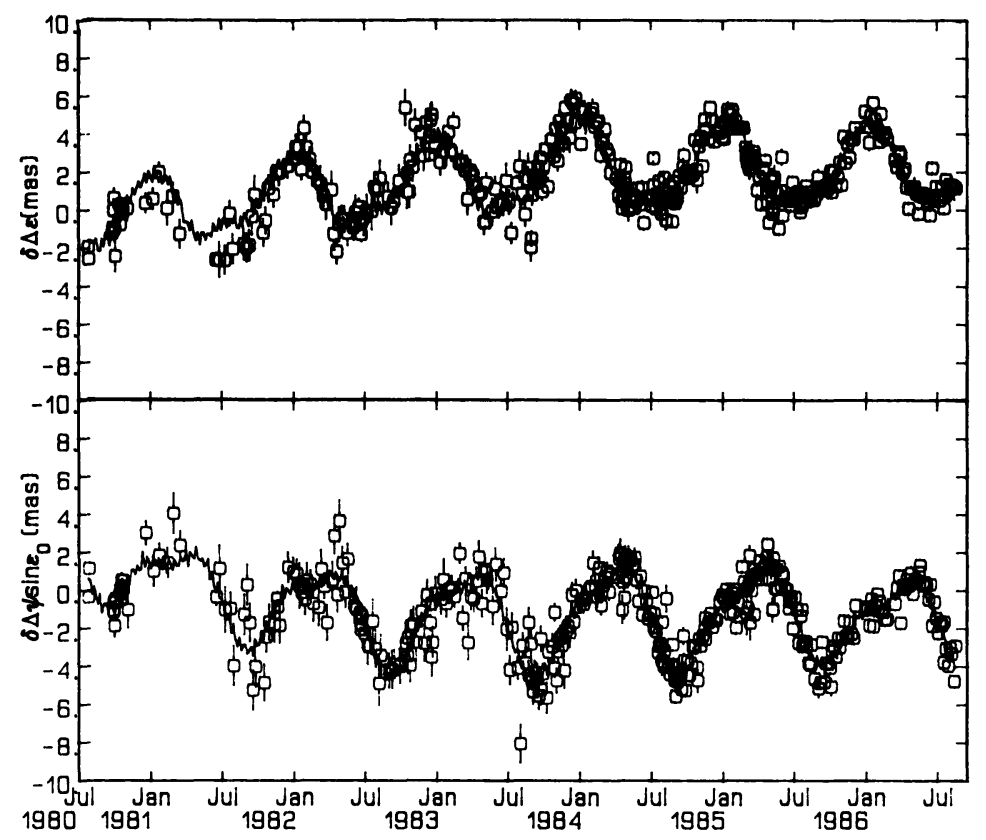

Figure 1. Estimates of $\delta \Delta \epsilon$ and $\delta \Delta \psi \sin \epsilon_{0}$ from the VLBI data analyzed in this paper. The solid line in each figure is computed from the corrections given in Table 1 and from long-period terms (periods $\geq 18.6$ years) which are not given in the table because their values are highly correlated and the estimates are not considered reliable.

might be considerably less than the bound given.

\section{CONCLUSION}

Our analysis has placed an upper bound of 0.6 mas ( $99.5 \%$ confidence limit) on the amplitude of the FCN. If the FCN is excited by surface loading with periods near one day, then this upper bound for the FCN amplitude combined with a lower bound for the damping time, allows an upper bound to be placed on the power-spectral density (PSD) of the surface loading. This upper bound for the surface loading is $0.06\left(\mathrm{gm} \mathrm{cm}^{-2}\right)^{2}$ day $(99.9 \%$ confidence limit) which is about five times smaller than the estimate by Eubanks et al. of the PSD of the surface loading due to atmospheric pressure variations. These results suggest that this PSD is dominated by noise or that the excitation of the FCN by surface loading is not as efficient as currently believed (Sasao and Wahr, 1981).

\section{ACKNOWLEDGMENTS}

This work was supported by the Air Force Geophysics Laboratory, contract F-19628-86-K-0025; NASA grant NAG5-538; and NSF grant EAR-83-06380. 


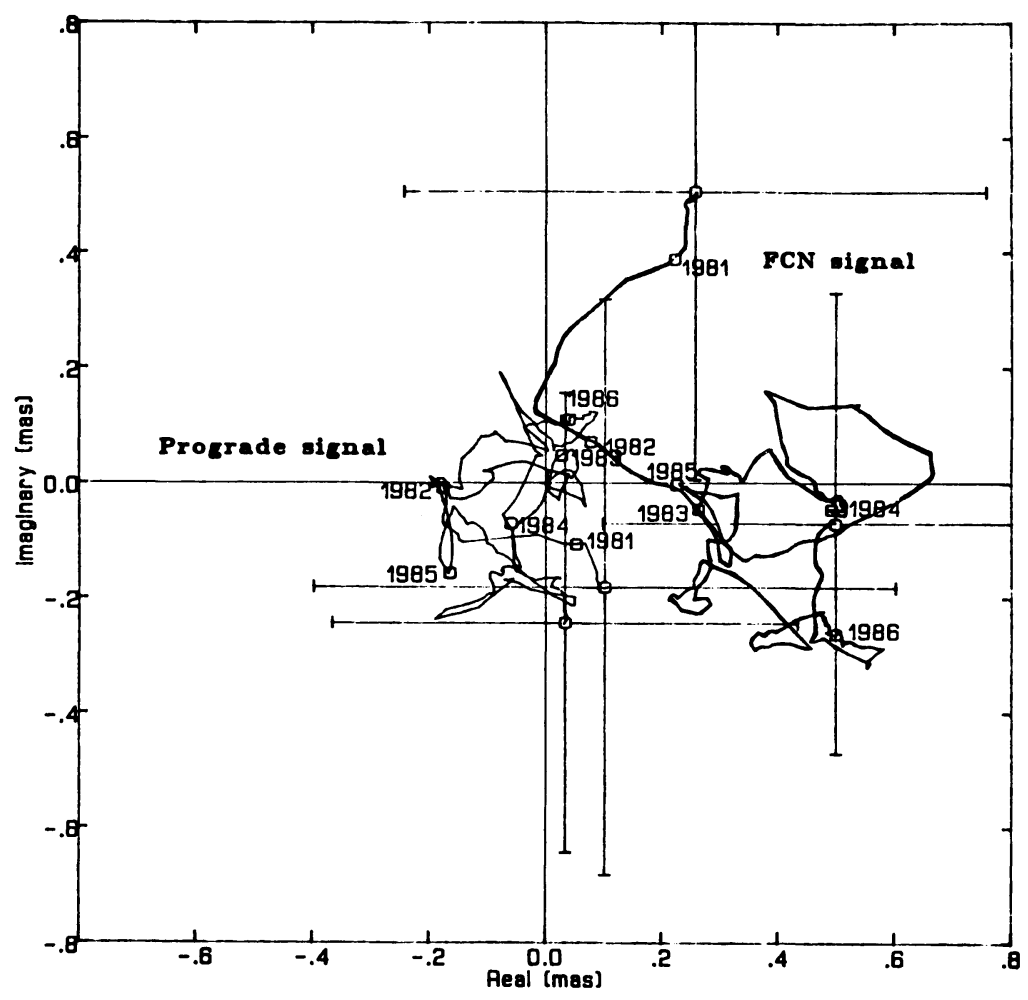

Figure 2. The estimates of the temporal changes in the complex amplitude of the FCN and the corresponding prograde terms, computed using the Kalman filter described in the text. The open squares show the values on January 1 for years between 1981 and 1986, as well as for the initial and final epochs for the data span. The error bars, shown only at the beginning and the end of the time interval spanned by the data, are one standard deviation, computed using the algorithm discussed in the caption of Table 1.

\section{REFERENCES}

Eubanks, T.M. et al., in "Proc. of the international conference on earth rotation and the terrestrial reference frame", Ohio State University, 326-340, 1985.

Gwinn, C.R. et al., EOS, 65, 859, 1984.

Gwinn, C.R. et al., J. Geophys. Res., 91, 4755-4765, 1986.

Herring, T.A. et al., EOS, 64, 674, 1983 .

Herring, T.A. et al., in "Proc. of the international conference on earth rotation and the terrestrial reference frame", Ohio State University, 307-325, 1985.

Herring, T.A. et al., J. Geophys. Res., 91, 4745-4754, 1986.

Liebelt,P.B., "An introduction to optimal estimation", Addison-Wesley, 273, 1967.

Sasao, T. and J.M.Wahr, Geophys. J. Roy. Astr. Soc., 64, 729-746, 1981.

Wahr, J.M. and T.Sasao, Geophys. J. Roy. Astr. Soc., 64, 747-765, 1981. 


\section{DISCUSSION}

Schub: Why have you not entered the 22 nutation coefficients as 'solve for' parameters in a global VLBI colution?

Roply by Herring: We haven't implemented the software yet to do that. We consider the nutation angle apprasch to be more flexible, as evidenced by our current paper. 